\title{
Reaction on a Change of User Context in Complex Mobile User Adaptive System
}

\author{
Ondrej Krejcar \\ VSB Technical University of Ostrava, Center for Applied Cybernetics, Department of \\ measurement and control, 17. Listopadu 15, 70833 Ostrava Poruba, Czech Republic \\ Ondrej.Krejcararemoteworld.net
}

\begin{abstract}
People life is today surrounded by a digital world environment which enables to interact with user and to react on user needs not only after user's requests, but also before the user needs grow. Paper deals with a concept of User Adaptive System (UAS) with two areas of user context along with reaction on them. A Predictive Data Push Technology (PDPT) Framework concept is mentioned as a possible solution fo user location context changes as well as a Biotelemetrical Monitoring System (BMS) is suitable for user biomedical data changes reaction on.
\end{abstract}

Keywords: User Adaptive System; Mobile Device; Biotelemetry; Prebuffering.

\section{Introduction}

The idea of User Adaptive Systems (UAS) grown from interaction between user and system (e.g. throws his mobile device). Such interaction can behold in the reaction on user's non declared requests. These requests are based on current user environment and biological or emotional state (e.g where I am?, what I feel?, am I ok?, etc.). Such user questions can be answered by sensors on user body or inside the user devices. By the help of user mobile device, we can get a user location (e.g. user current position, user future-predicted position, his movement and tracking, etc.). Biomedical sensors on user body can detect several important biomedical data, which can be used for determination of user emotional state in the environment around.

By the combination of user's requests (known or predicted) in conjunction with other sources of user's knowledge and behaviors, the sophisticated information system can be developed as UAS. The impact of UAS can be seen in the increased user comfort when accessing these mobile UAS. In ideal case, everything what user can imagine to have in his mobile UAS is there.

A one specific kind of problems is based in increased data amount in new mobile systems. In current cases, the user need to specify a data to be downloaded to his mobile device and he need to wait for data downloading and displaying. Due to a several limitations in hardware of current mobile devices, the use of such large amount data has result in lower user comfort. The needs of any techniques to reduce such large data amount or to preload them before user's needs, is still growing up. We created a Predictive Data Push Technology (PDPT) Framework to solve these 
problems by data prebuffering. Our idea can be applied on a variety of current and future wireless network systems. More usability of PDPT grows from definition of area to be prebuffered as well as from evaluation of artifacts or other user's behavior sources. Additional will be presented in sections [2], [3].

The second area of problems which we would like to solve is based on a users biomedical data inputs and a wide area of their possible utility. Current body sensors allow a monitoring of a huge number of biomedical data information (e.g. use a special t-shirt equipped with an ECG, temperature, pressure or pulse sensors). Current hitech mobile devices are equipped with a large scale display, provide a large memory capabilities and a wide spectrum of network standards plus embedded GPS module (e.g. HTC Touch HD, HD2). These devices have built-in also a special accelerometer which can be used to determine a user's body situation (user is staying or lying). Last but not least equipment is a light sensor which can be used not only to brightness regulation.

\section{Reaction on a Change of Location}

We can imagine the usage of such described UAS in the information systems area of botanical or zoological gardens. In such areas there has been a big potential of usage of a continual localization by use of GPS or wireless networks (in case the GPS has not a sufficient signal - e.g. in urban centers or neighborhoods with high buildings, forest parks or in deep valleys). There is also a possibility to compute a current and predicted user track, so we can predict a position of user in near future (e.g. 25 meters north in one minute). Usability of these information sources is uncountable.

One of possible use of user predicted position is for a determination of a data, which will be needed by user of mobile UAS in near future. Such data (data artifacts) can be preloaded to user's device memory for future requests. The need of preloaded artifacts grown from a need of up to date data context of dynamic online system. Of course when static offline system is used, there is a possibility to load a needed data before usage (e.g store artifacts at SD Card with a size limit to several GB). When user request info about his location in context of zoo or garden (turn-on the device is only needed by user), the client application will respond with a map of near surroundings and a prebuffered data artifacts. User can select a documentary about animals or vegetation around him which can be viewed or played. User can act with direct requests to selected kinds of these. These preferred kinds will be taken into account to evaluate future objects/artifacts and preloaded only the most important ones for a user. The type of artifact is also evaluable as well as his size because the user may not want to look at too long or micro presentation. From several statistic info obtained from users tracks, the most frequented ways in gardens can be also found. By the help of some mentioned info sources a very sophisticated dynamical area definition can be developed.

As client devices of online UAS, the mobile wireless devices like PDA or Smart phones are commonly used with an internet connection. The connection speed of the two most common standards GPRS and WiFi varies from hundreds of kilobits to several megabits per second. In case of online UAS or some other types of facility management, zoological or botanical gardens, libraries or museums information 
systems, the WiFi infrastructure network is often used to connect mobile device clients to a server. Unfortunately, the low performance hardware components are used in PDAs or SmartPhones due to a very limited space. By this fact a theoretical maximum connection speed is not achievable on these devices. The limited connection speed represents a problem for online systems using large artifacts data files. It is not possible to preload these artifacts before the mobile device is used in a remote access state. This problem was found as a very important. The goal is to combine the data networking capabilities of RF wireless LANs [4], [5] with accurate user location and tracking capabilities for user needed data prebuffering. These properties are used as an information base for an extension of existing UAS or to create special new ones. Information about location is used to determine an actual and a future position of a user. The following section describes also the conceptual and technical details about PDPT Framework.

\section{Reaction on a Change of Biomedical Data}

We are using principles of UAS in area of biomedical data processing, where we try to predict some kind of problems by patient data analysis. We developed a contextaware Biotelemetrical Monitoring System (BMS) [6] as a part of the UAS and PDPT Framework project facilitates the following:

- Real-time collection of the patient vital signs (e.g. ECG, EEG) by means of a Body Area Network (BAN) or direct wireless connection to PDA device monitoring station.

- Real-time transmission of data using the wireless connectivity to the healthcare professionals through a complete architecture including a server database, web services, doctors web access to patients collected and preprocessed data.

- Seamless handover over different wireless communication technologies such as BlueTooth, WiFi, GPRS or UMTS.

- Context-aware infrastructure to sense the context (e.g. location, availability, activity, role) of the patients and Emergency Response Services (ERSs) to provide assistance to the patient in case of an emergency. An ERS could be fixed (e.g. hospital) or mobile (e.g. caregiver). A mobile ERS is published in the BMS.

Classical access to patients request are made by reactive flowchart, where a patient is equipped with a classical offline measuring devices with some type of alarms. Every violated alarm need to be a carried out by doctor decision. Such access is very timeconsuming. Second proposed access is based on a proactive principle, where the patient is equipped with an online measuring devices with an online connection to some kind of superior system (in our case the BMS is presented). In this case, a patient's measured data are processed on mobile monitoring station or at server. An alert will invoke when the anomaly data are founded in patient's records. Consequently the doctor is responsible to make a decision to invoke other ERSs or to remove Alarm (in case of false detection of anomaly). Such kind of behavior is based on UAS. In many of events a predicted and solved problems can save a life. The predicted patient's problems are in most cases minor in compare to a major problems detected in time where occurred. 


\section{Conclusions}

A two main directions of UAS as a reaction a change of location and reaction on a change of biomedical data were described with main focus on use of mobile devices. Coexistence of proposed solutions is in unnumbered areas and the results of complex solution are better than expected. The developed UAS can be stocked on a wide range of wireless mobile devices for its main issue at increased downlink speed. The localization part of PDPT framework is currently used in another project of biotelemetrical system for home care agencies to make a patient's life safer [6]. Several areas for PDPT stocking was founded in projects of hydro-information system "Transcat" [1], [2]. Several of UAS principles can be used there also. The last but not least was found in social dimension of UAS where the focus is now moved from explicit to implicit collaboration [7]. These possibilities will be investigated in future.

Acknowledgement. This work was supported by the Ministry of Education of the Czech Republic under Project 1M0567.

\section{References}

1. Horak, J., Unucka, J., Stromsky, J., Marsik, V., Orlik, A.: TRANSCAT DSS architecture and modelling services. Journal: Control and Cybernetics 35, 47-71 (2006)

2. Horak, J., Orlik, A., Stromsky, J.: Web services for distributed and interoperable hydroinformation systems. Journal: Hydrology and Earth System Sciences 12, 635-644 (2008)

3. Krejcar, O.: Problem Solving of Low Data Throughput on Mobile Devices by Artefacts Prebuffering. EURASIP Journal on Wireless Communications and Networking, Article ID 8025238 (2010)

4. Brida, P., Duha, J., Krasnovsky, M.: On the accuracy of weighted proximity based localization in wireless sensor networks. In: Personal Wireless Communications. IFIP, vol. 245, pp. 423-432 (2007)

5. Krejcar, O.: Large Multimedia Artifacts Prebuffering in Mobile Information Systems as Location Context Awareness. In: 4th International Symposium on Wireless Pervasive Computing, ISWPC 2009, Melbourne, Australia, February 11-13, pp. 216-220 (2009), doi:10.1109/ISWPC.2009.4800591

6. Krejcar, O., Janckulik, D., Motalova, L.: Complex Biomedical System with Biotelemetric Monitoring of Life Functions. In: Proceedings of the IEEE Eurocon 2009, St. Petersburg, Russia, May 18-23, pp. 138-141 (2009), doi:10.1109/EURCON.2009.5167618

7. Longo, L., Barrett, S., Dondio, P.: TOWARD SOCIAL SEARCH From Explicit to Implicit Collaboration to Predict Users' Interests. In: WEBIST 2009, 5th International Conference on Web Information Systems and Technologies Inst. Syst. \& Technol. Informat., Control \& Commun., Lisbon, Portugal, March 23-26, pp. 693-696 (2009) 